\title{
Voltage Stability Assessment in a Power System Using Singular Value Decomposition Technique
}

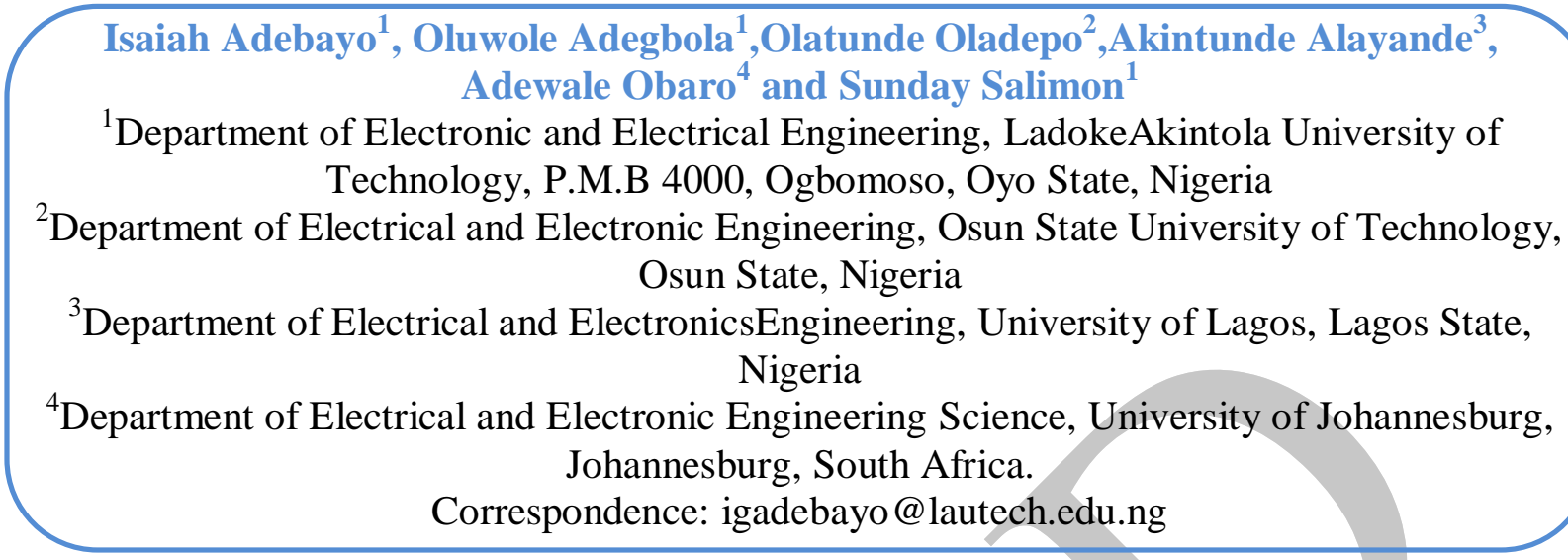

Abstract

Voltage instability, which is the principal factor behind blackouts experienced in some developing and developed countries throughout the globe, has posed a great threat and concern to both utilities and end-users of electricity. As a result, there is the need to identify weak buses that are susceptible to voltage collapse in other to take proper measures to forestall the eventual occurrence of blackout. Hence, this paper aims to utilize singular value decomposition method to identify buses in critical state near collapse in a power system. The singular eigenvalue of the reduced matrix is also computed and the corresponding eigenvectors are also determined. The L-Index approach is also adopted for identification of buses near collapse in a power system in order to verify results obtained using the SVD method. The Nigerian 28-bus and the IEEE 14-bus power systems are used to evaluate the viability of all the presented methods. The simulated results show the effectiveness of the SVD approach in identifying critical buses close to the point of voltage collapse.

Keywords: Voltage instability, power system, voltage collapse, critical bus, singular value decomposition

\section{Introduction}

One of the most important factors to consider when planning and operating a power system is the issue of voltage stability. Unfortunately, voltage stability problems have caused quite a bit of power outages experienced in both developed and developing countries. For example, the horrific power outage in Tokyo in 1987, Britain, Sweden, Denmark, Canada and the United States were all attributed to the root cause of voltage instability. Millions of electricity consumers were affected by these shutdowns [1] - [3]. Voltage instability is one of the main and important problems among the various factors that can lead to power system outages. When the power system is overloaded, the voltage becomes unstable because the generating power sources cannot generate enough reactive power [4]. Therefore, voltage stability is an important issue to consider in the planning and operation of power systems. The ability of a power system to keep all bus voltages within standard tolerances after being subjected to a disturbance is called voltage stability. These disturbances include a sudden or gradual increase in load demand or a change in system state that results in a gradual and irresistible drop in voltage [5].

Based on the aforementioned challenge, system operators need to know the mechanisms that cause voltage instability. For this reason, many researchers have used various methods to carry out various studies for evaluating the voltage stability of power systems. Some of these techniques include continuous power flow (CPF), multiple power flow solutions that use sensitivity analysis, PV and PQ curves, and optimization-based methods and so on. Some of these techniques have their strengths and 
weaknesses. For example, the evaluation of voltage stability using sensitivity analysis techniques is suitable for simple power supply systems, but when applied to multi-machine systems, there may be discriminant errors [6]. According to the literature, PV and PQ curve techniques are feasible for assessing voltage stability, but have the drawback of consuming enormous amounts of time and complexity in large power systems [7]. Also, sufficient physical information is not provided due to stability issues when using PVQV curves [8]. Therefore, there is a need for appropriate techniques that can be used to analyze the voltage stability of power systems.

Recently, obtaining the solution to static voltage stability problem using voltage stability indices has attracted attention of researchers. Voltage stability indices are very useful in restoring the stability of a power system with the scalar magnitude used to monitor changes in system parameters. Besides, the indices can provide give ideas of margins for specific operating points from critical points where voltage collapse can occur [9] [10]. The L-Index, voltage collapse proximity index (VCPI), Fast Voltage Stability Index (FVSI), Line Stability Factor (LQP), power stability index and voltage deviation index [10]-[14] are few examples of these indices. These indices have proven to be effective and reliable in voltage stability analysis.

[15] reported the evaluation of voltage stability using modal analysis of SVD (Singular Value Decomposition) and modal analysis of the reduced Jacobian methods. These indices can efficiently identify weak nodes on the verge of voltage collapse. Therefore, in this paper, the performance of these two indices was compared and tested on the Nigerian 28bus and IEEE 14bus power supply systems.

In the next section, the mathematical formulation for the SVD and LIndex approaches are presented. The simulation results obtained are presented and discussed in Section III. Section IV is the conclusion.

\section{Problem Formulations}

The derivations of the SVD and L-index are given under this section.

A. Singular Value Decomposition Technique

First, the load flow solution for the steady state operation of the system is obtained using the Newton Raphson technique (1)

$\left[\begin{array}{l}\Delta P^{(k)} \\ \Delta Q^{(k)}\end{array}\right]=J^{(k)}\left[\begin{array}{l}\Delta \theta^{(k)} \\ \Delta U^{(k)}\end{array}\right]$

where

$\left[\Delta P^{(k)}\right]=P-\Delta P^{(k)}(2)$

$\left[\Delta Q^{(k)}\right]=Q-\Delta Q^{(k)}(3)$

$\Delta P$ and $\Delta Q$ are the mismatch vectorsof real and reactive powers; $\Delta U$ and $\Delta \theta$ represent the unknown voltage magnitude and angle correction vectors, respectively; $k$ and $J$ depict the number of iterations and full Jacobian matrix, respectively.

The full Jacobian matrix is can be expressed

$J=\left[\begin{array}{ll}J^{P \theta} & J^{P U} \\ J^{Q \theta} & J^{Q U}\end{array}\right]$

The comprehensive details of the elements of equation (4) are found in [5].

The change in reactive power $\Delta Q$ is established on the basis of the fact that the voltage magnitude of a power system is mostly due to shortage of reactive power while change in active power, $\Delta P$ is taken to be zero. The reduced Jacobian matrix substitution of these facts in equation (1) yields:

$\left[\begin{array}{c}0 \\ \Delta Q\end{array}\right]=\left[\begin{array}{cc}J^{P \theta} & J^{P U} \\ J^{Q \theta} & J^{Q U}\end{array}\right]\left[\begin{array}{c}\Delta \theta \\ \Delta U\end{array}\right]$

$\Delta Q=\left(J^{Q U}-J^{Q \theta}\left(J^{-1}\right)^{P \theta} J^{P U}\right) \Delta U=J_{T} \Delta U$

where $J_{T}=\left(J^{Q U}-J^{Q \theta}\left(J^{-1}\right)^{P \theta} J^{P U}\right)(7)$ 
From equation (1),

$\Delta U=J_{T}{ }^{-1} \Delta Q(8)$

The above formulated equations are used to establish the SVD equations. For a real square matrix $B$ of $N \times N$ dimension, the SVD is expressed as:

$B=F \lambda H^{T}=\sum_{k=1}^{N} \varepsilon_{k} f_{k} h_{k}^{T}(9)$

where $\varepsilon_{k}$ is the minimum singular value. $\varepsilon_{k} \geq 0$ for all k. Also, $F$ and $H$ are the $N \times N$ orthogonal matrices, $f_{i}$ and $h_{i}$ are the left and right singular vectors respectively and $\lambda$ is a diagonal matrix with :

$\sum B=\operatorname{diag}\left[\varepsilon_{k}(B)\right] \quad i=1,2,3, \ldots . . N$

If the SVD is applied to the reduced Jacobian matrix of equation (7), then we have:

$J_{T}=F \lambda H^{T}=\sum_{k=1}^{N} \varepsilon_{k} f_{k} h_{k}^{T}$

The closeness of the singularity of the Jacobian is denoted by the least singular value $\varepsilon_{k}\left(J_{R}\right)$. In the case of a disturbance stand point, let

$$
\left[\begin{array}{c}
\Delta P=0 \\
\Delta Q
\end{array}\right]=f_{M}
$$

and

$$
\left[\begin{array}{c}
\Delta \theta \\
\Delta U
\end{array}\right]=\varepsilon_{M}^{-1} h_{M}
$$

where $f_{M}$ and $h_{M}$ are the last columns of $\mathrm{F}$ and $\mathrm{H}$ respectively.

Based on the established equation, the following rules can be set [5]:

(i) The steady-state stability limit is displayed by the minimum singular value.

(ii) The right singular vector corresponding to the smallest singular value indicates the most sensitive voltage.

Therefore, it can be inferred that the direction most sensitive to changes in active and ineffective power injections is represented by the left singular vector corresponding to the minimum singular value [5].

\section{B. L-Index Approach}

The L-index represents a critical part in assessing the distance to the stability limit in the actual state of the power system. It also defines the stability of the entire system. As given in [10]:

$$
L_{j}=\left|1-\sum_{i=1}^{n} F_{j i} \frac{U_{i}}{U_{j}}\right|(1
$$

where $\mathrm{U}$ is the voltage at the node. The subscripts $j$ and $i$ are the load and generator bus numbers respectively. $F_{j i}$ is an element in sub-matrix $F_{L G}$ as presented in the expression of equation (15).

$$
\left|\begin{array}{l}
U_{L} \\
I_{G}
\end{array}\right|=\left|\begin{array}{ll}
Z_{L L} & F_{L G} \\
T_{G L} & Y_{G G}
\end{array}\right|\left|\begin{array}{l}
I_{L} \\
U_{G}
\end{array}\right|(15)
$$

where

$$
F_{L G}=-\left|Y_{L L}\right|^{-1}\left|Y_{L G}\right|(16)
$$

Detailed definitions of the parameters can be found in [16]

By interpretation, equation (14) implies that if the L-Index value is close to zero, it shows that the power system has a stable voltage maximum margin. On the other hand, if the value of $L_{j}$ is close to 1 , this indicates that the system is almost unstable in voltage. 


\section{Results and discussion}

The efficiency of the presented methods is tested on the Nigerian 28-bus and the IEEE 14-bus electrical systems. The Nigerian 28-bus power system consists of nine generator buses and nineteen load buses with thirty-two transmission lines. Likewise, the IEEE 14-bus power system consists of five generator buses and nine load buses with twenty transmission lines. MATLAB R (2015a) was used for all simulations performed. The singular values (for the Nigerian 28-bus test system) of the reduced Jacobian matrix of equation (11) are calculated and the results obtained indicate that the system is voltage stable because of all the values obtained are uniformly positive.

Nineteen special values are calculated for Nigerian $330-\mathrm{kV}$ grid system. Of all these values, the most dominant one is shown in Figure 1. Tables 1 and 2 also show the two smallest singular values and five randomly selected load buses of the 28-vehicle electrical system. bus and their respective single vectors. These load buses are chosen because of the larger value of the single vectors they display.

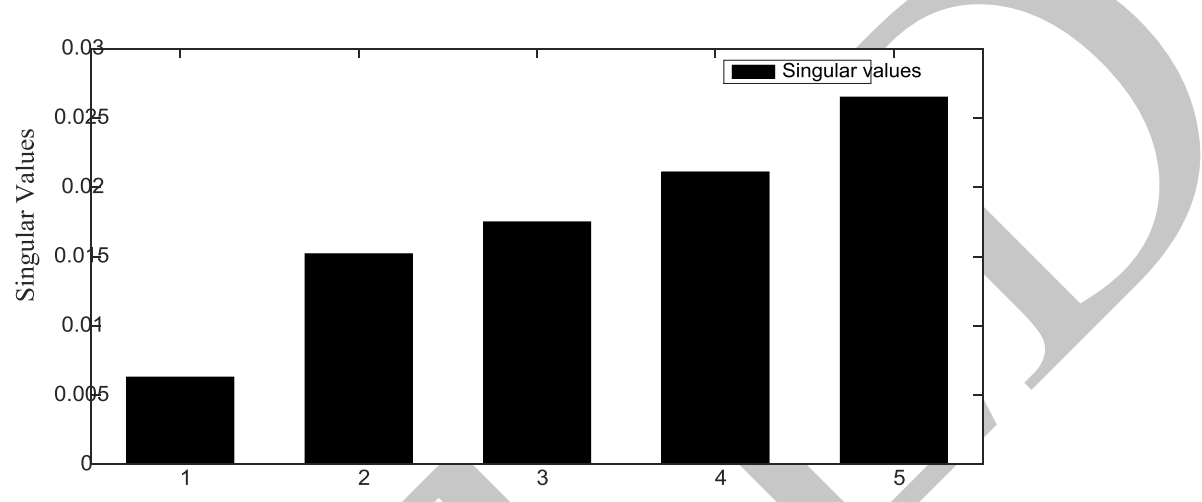

Figure 1: Singular value of Nigeria's 28-bus power system

Table 1: Singular vector corresponding to the smallest singular value of the Nigerian 28 bus

\begin{tabular}{|c|c|c|c|}
\hline First Singular Value $\mathbf{= 0 . 0 0 6 3}$ & \multirow{2}{*}{ Voltage Mag. (p.u) } \\
\cline { 1 - 3 } Ranking order & $\begin{array}{c}\text { Bus Singular } \\
\text { Vector }\end{array}$ & Bus Number & 0.8698 \\
\hline $1^{\text {st }}$ & 0.9514 & 16 & 0.9122 \\
\hline $2^{\text {nd }}$ & 0.2587 & 19 & 0.9023 \\
\hline $4^{\text {th }}$ & 0.0962 & 22 & 0.8702 \\
\hline $5^{\text {th }}$ & 0.0155 & 13 & 0.9867 \\
\hline $3^{\text {rd }}$ & 0.1169 & 25 & \\
\hline
\end{tabular}

Table 2: Singular vector corresponding to the second singular value

\begin{tabular}{|c|c|c|c|}
\hline \multicolumn{2}{|c|}{ Second Singular Value $=\mathbf{0 . 0 1 5 2}$} \\
\hline $\begin{array}{c}\text { Ranking } \\
\text { Order }\end{array}$ & $\begin{array}{l}\text { Bus Singular } \\
\text { Vector }\end{array}$ & $\begin{array}{c}\text { Voltage } \\
\text { Magnitude (p.u) }\end{array}$ & Bus Number \\
\hline $1^{\text {st }}$ & 0.7830 & 0.8702 & 13 \\
\hline $5^{\text {th }}$ & 0.0167 & 0.9122 & 19 \\
\hline $3^{\text {rd }}$ & 0.0255 & 0.9023 & 22 \\
\hline $2^{\text {nd }}$ & 0.0332 & 0.8698 & 16 \\
\hline $4^{\text {th }}$ & 0.0245 & 0.9867 & 25 \\
\hline
\end{tabular}

It can be seen from Table 1 that bus 16 was chosen as the best with the maximum value $(0.9514)$ of the singular eigenvector. Therefore, the load bus 16 is identified as a critical bus of Nigerian28-bus power system or a bus prone to voltage instability. The results obtained using the L-Index method are shown in Table 3 . Bus 16 of the selected load bus $(13,16,19,22$ and 25) are considered system weak buses because it have a maximum value of L-Index (0.2000). 
DOI : https://dx.doi.org/10.26808/rs.ed.i11v5.03

International Journal of Emerging Trends in Engineering and Development

Issue 11, Vol.5 (Aug-Sep 2021)

Available online on http://www.rspublication.com/ijeted/ijeted_index.htm

ISSN 2249-6149

Table 3: Nigeria 28-bus Power System L-Index Results

\begin{tabular}{|c|c|c|}
\hline Bus Number & L-Index & Voltage Mag. (p.u) \\
\hline 13 & 0.1423 & 0.8702 \\
\hline 16 & 0.2000 & 0.8698 \\
\hline 19 & 0.0966 & 0.9122 \\
\hline 22 & 0.1259 & 0.9023 \\
\hline 25 & 0.0629 & 0.9867 \\
\hline
\end{tabular}

In addition, for IEEE 14 bus power systems, singular eigenvectors and eigenvalues were calculated. Since the singular values obtained are all positive numbers, the system (IEEE 14-bus power system) has a good voltage stability. The corresponding bus singular vector, knowing that the first minimum eigenvalue is 3.0900 , is also calculated as shown in Table 4. The second minimum value and its corresponding eigenvector are also calculated as shown in Table 5.

Table 4: IEEE 14-bus system first singular value and corresponding singular vector

\begin{tabular}{|c|c|c|}
\hline \multicolumn{3}{|c|}{ First minimum Singular Value $=\mathbf{3 . 0 9 0 0}$} \\
\hline Ranking order & Bus Number & Bus Singular Vector \\
\hline $8^{\text {th }}$ & 6 & 0.1163 \\
\hline $4^{\text {th }}$ & 7 & 0.3629 \\
\hline $9^{\text {th }}$ & 8 & 0.0838 \\
\hline $3^{\text {rd }}$ & 9 & 0.3877 \\
\hline $2^{\text {nd }}$ & 10 & 0.4486 \\
\hline $5^{\text {th }}$ & 11 & 0.3194 \\
\hline $7^{\text {th }}$ & 12 & 0.1607 \\
\hline $6^{\text {th }}$ & 13 & 0.1960 \\
\hline $1^{\text {st }}$ & 14 & $\mathbf{0 . 5 7 4 4}$ \\
\hline
\end{tabular}

Table 5: Singular vector corresponding to the second singular value of IEEE14-bus system

\begin{tabular}{|c|c|c|}
\hline \multicolumn{3}{|c|}{ Second minimum Singular Value $\mathbf{5 . 5 7 4 8}$} \\
\hline Bus Number & Bus Singular Vector & Voltage Mag. (p.u) \\
\hline 6 & 0.0656 & 1.0600 \\
\hline 7 & 0.1726 & 1.0450 \\
\hline 8 & 0.0510 & 1.0100 \\
\hline 9 & 0.1792 & 1.0700 \\
\hline 10 & 0.3508 & 1.0900 \\
\hline 11 & 0.3699 & 1.0185 \\
\hline 12 & 0.5701 & 1.0485 \\
\hline 13 & 0.3952 & 1.0096 \\
\hline 14 & 0.4360 & 1.0064 \\
\hline
\end{tabular}

It can be seen from Table 4 that the smallest singular value with the largest singular vector (0.5744) is connected to the load bus (14) of the IEEE14-bus power system. Therefore, the load bus 14 is ranked highest and considered as a critical bus in the system where the voltage is prone to instability. For ease of display, the singular vector corresponding to each load bus in the system is also shown in the form of a graph in Figure 2.

Each load bus L-Index is also calculated as shown in Figure 3. The bus system with the highest Lindex value is considered the weakest bus. As shown in Figure 3, that load bus 14 has the highest value of the L-index and is thus taken as the weak bus of the IEEE 14-bus power system. This confirms the result of the singular value decomposition method. Figure 4 is a graphical representation of the comparison of the two methods utilized in this paper. 


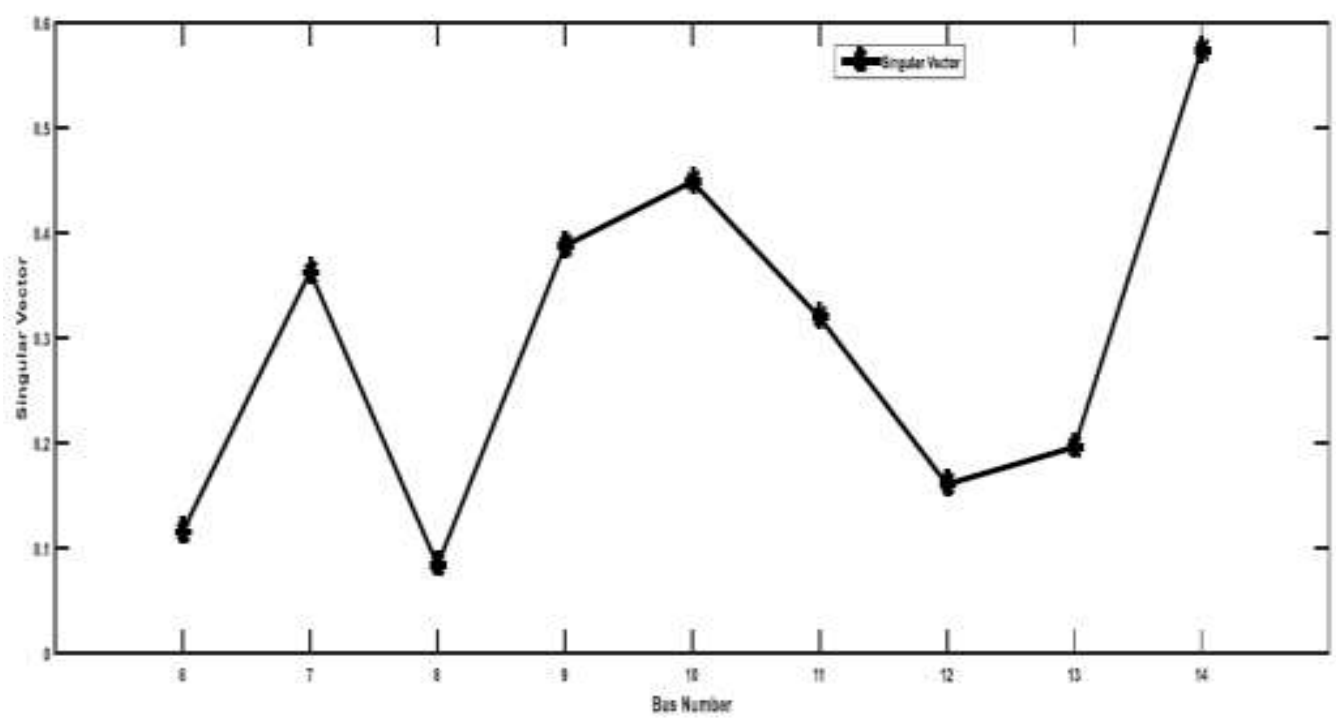

Figure 2: Singular vectors of the IEEE 14-bus power system

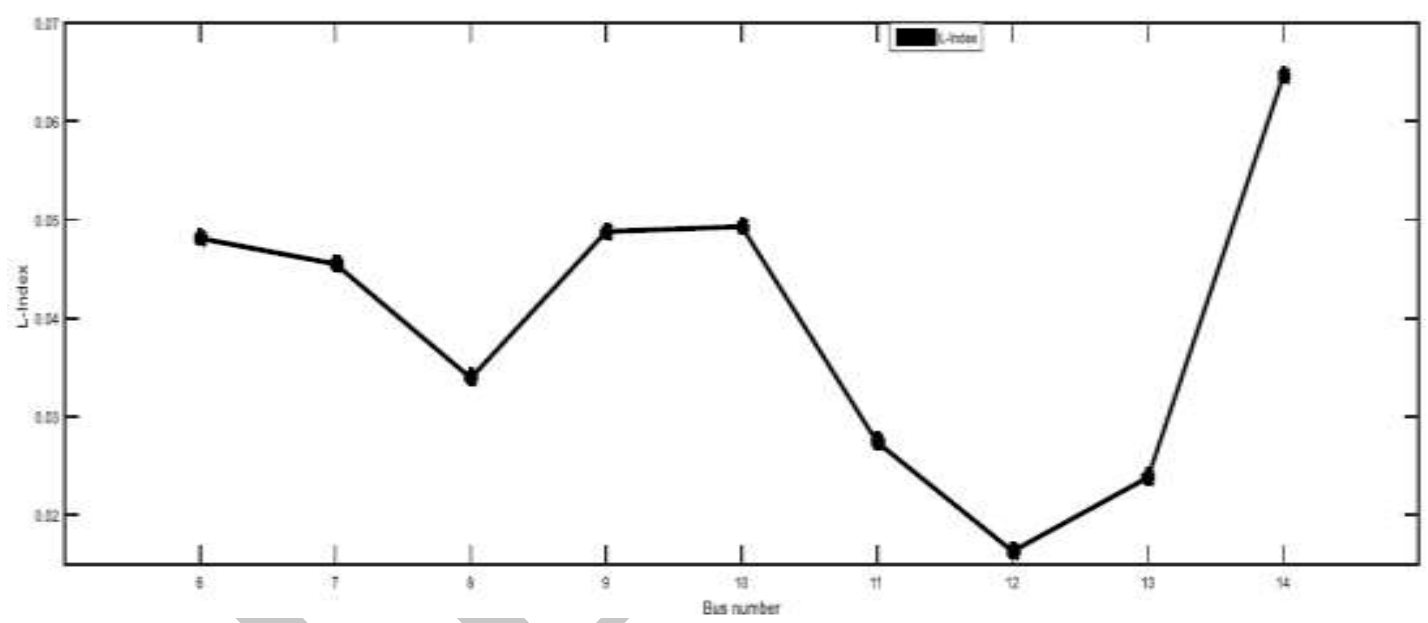

Fig. 3: Graphical representation of the L-Index of the IEEE 14-bus system

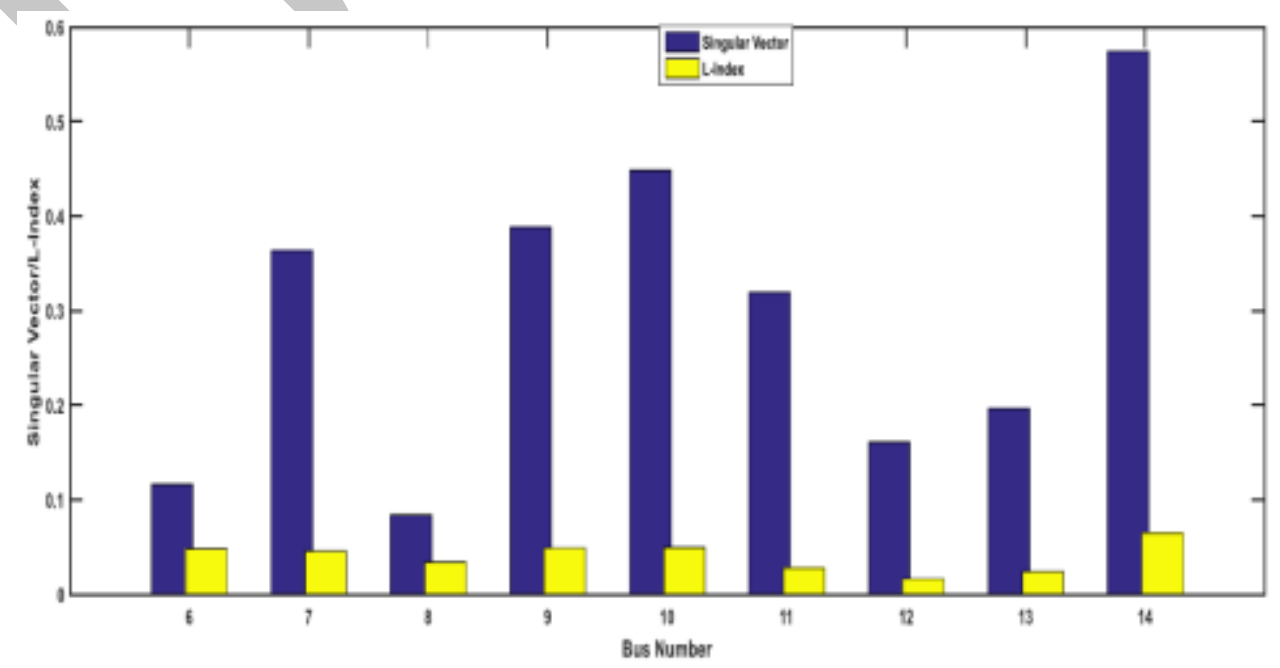

Figure 4: Comparison of IEEE 14-bus system singular value and L-Index 


\section{Conclusion}

The effectiveness of using both the SVD and L-Index methods to identify critical buses in the power system has been fully demonstrated in this work. All simulations are performed using MATLAB R (2015a). The effectiveness of the presented methodology has been tested on both Nigerian 28-bus and IEEE 14-bus power systems. The results obtained show that both techniques can be used to easily identify vulnerable load buses with voltage instability. The results of this study can be of great help in during operations and planning of power system.

\section{References}

[1] Ohno, T.; Imai, S. The 1987 Tokyo blackout. In Proceedings of the 2006 IEEE PES Power Systems Conference and Exposition, Atlanta, GA, USA, 29 October-1 November 2006; pp. 314-318.

[2] Final Report on the August 14, 2003 Blackout in the United States and Canada: Causes and Recommendations. Available online: https://www.osti.gov/etdeweb/biblio/20461178 (accessed on 23 April 2019).

[3] Mir Sayed Shah Danish , TomonobuSenjyu , Sayed Mir Shah Danish , NajibRahmanSabory, Narayanan K and Paras Mandal. A Recap of Voltage Stability Indices in the Past Three Decades. Energies 2019, 12, 1544; doi:10.3390/en12081544

[4] Sindy L. Ramírez-P, Carlos A. Lozano-M and Nayiver G. Caicedo-D. Review and Classification of Indices for Voltage Stability Monitoring using PMU Measurements. Journal of Engineering Science and Technology Review 11 (4) (2018) 180 - 198.

[5] K. Ellithy, M. Shaheen, M. Al-Athba A. Al-Subaie, S. Al-Mohannadi, S. Al-Okkah, S. Abu-Eidah. Voltage Stability Evaluation of Real Power Transmission System Using Singular Value Decomposition Technique. 2nd IEEE International Conference on Power and Energy (PECon 08), December 1-3, 2008, Johor Baharu, Malaysia.

[6] Jianhong Pan, Aidi Dong, Jiashu Fan, and Yang Li, Online Static Voltage Stability Monitoring for Power Systems Using PMU Data. Hindawi Mathematical Problems in Engineering Volume 2020, Article ID 6667842, 8 pages https://doi.org/10.1155/2020/6667842

[7] I. G. Adebayo, A. A. Jimoh and A. A. Yusuff, "Prediction of voltage collapse through voltage collapse proximity index and inherent structural characteristics of power system," 2015 IEEE PES Asia-Pacific Power and Energy Engineering Conference (APPEEC), 2015, pp. 1-5, doi: 10.1109/APPEEC.2015.7381025.

[8] H.H. Goh, Q.S. Chu, S.W. Lee, B.C. Kok, K.C. Goh, K.T.K. Teo, Evaluation for Voltage Stability Indices in Power System Using Artificial Neural Network. International Conference on Sustainable Design, Engineering and Construction, Procedia Engineering 118 ( 2015 ) 1127 1136

[9] Lof P-A, Andersson G, Hill DJ. Voltage stability indices for stressed power systems. IEEE Trans Power Syst 1993;8(1):326-35.

[10] Kessel, P.; Glavitsch, H. Estimating the Voltage Stability of a Power System. IEEE Trans. Power Deliv. 1986, 1,V346-354.

[11] Musirin, I.; Rahman, T.K.A. On-line voltage stability based contingency ranking using fast voltage stability index (FVSI). In Proceedings of the IEEE/PES Transmission and Distribution Conference and Exhibition, Yokohama, Japan, 6-10 October 2002; Volume 2, pp. 1118-1123

[12] Balamourougan, V.; Sidhu, T.S.; Sachdev, M.S. Technique for online prediction of voltage collapse. IEE Proc. Gen. Transm. Distrib. IET 2009, 151, 453-460. 
[13] Aman, M.M.; Jasmon, G.B.; Mokhlis, H.; Bakar, A.H.A. Optimal placement and sizing of a DG based on a new power stability index and line losses. Int. J. Electr. Power Energy Syst. 2012, 43, 1296-1304.

[14] Yang, C.F.; Lai, G.G.; Lee, C.H.; Su, C.T.; Chang, G.W. Optimal setting of reactive compensation devices with an improved voltage stability index for voltage stability enhancement. Int. J. Electr. Power Energy Syst. 2012, 37, 50-57.

[15] M. K. Jalboub, H. S. Rajamani, R.A. Abd-Alhameed and A. M. Ihbal. Weakest Bus Identification Based on Modal Analysis and Singular Value Decomposition Techniques. Iraq J. Electrical and Electronic Engineering, Vol.7 No.2, 2011.

[16] I. G. Adebayo and Y. Sun, "Application of Network Structural Properties of a Power System for Voltage Stability Assessment in an Electric Power Grid," 2021 International Conference on Sustainable Energy and Future Electric Transportation (SEFET), 2021, pp. 1-5, doi: 10.1109/SeFet48154.2021.9375653. 